\title{
Heating Techniques for an EBT Reactor
}

\author{
N. A. U'ckan \\ D. B. Batchelor
}

\section{OAK RIDGE NATIONAL LABORATORY} OPERATED BY UNION CARBIDE CORPORATION - FOR THE DEPARTMENT OF ENERGY 


\section{DISCLAIMER}

This report was prepared as an account of work sponsored by an agency of the United States Government. Neither the United States Government nor any agency Thereof, nor any of their employees, makes any warranty, express or implied, or assumes any legal liability or responsibility for the accuracy, completeness, or usefulness of any information, apparatus, product, or process disclosed, or represents that its use would not infringe privately owned rights. Reference herein to any specific commercial product, process, or service by trade name, trademark, manufacturer, or otherwise does not necessarily constitute or imply its endorsement, recommendation, or favoring by the United States Government or any agency thereof. The views and opinions of authors expressed herein do not necessarily state or reflect those of the United States Government or any agency thereof. 


\section{DISCLAIMER}

Portions of this document may be illegible in electronic image products. Images are produced from the best available original document. 
Printed in the United States of America. Available from National Technical Information Service

U.S. Department of Commerce

5285 Port Royal Road, Springfield, Virginia 22161

Price: Printed Copy $\$ 4.50$; Microfiche $\$ 3.00$

This report was prepared as an account of work sponsored by an agency of the United States Government. Neither the United States Government nor any agency thereof, nor any of their employees, contractors, subcontractors, or their employees, makes any warranty, express or implied, nor assumes any legal liability or responsibility for any third party's use or the results of such use of any information, apparatus, product or process disclosed in this report, nor represents that its use by such third party would not infringe privately owned rights. 
Contract No. W-7405-eng-26

FUSION ENERGY DIVISION

N. A. Uckan

D. B. Batchelor

Date Published - May, 1978

Prepared by the

OAK RIDGE NATIONAL LABORATORY

Oak Ridge, Tennessee 37830

operated by

UNION CARBIDE CORPORATION

for the

DEPARTMENT OF ENERGY 


\section{THIS PAGE}

\section{WAS INTENTIONALLY \\ LEFT BLANK}


CONTENTS

ABSTRACT ......................... . $v$

1. INTRODUCTION . . . . . . . . . . . . . . . . . . 1

2. MICROWAVE HEATING OF AN EBT REACTOR . . . . . . . . . . 2

3. NEUTRAL BEAM HEATING OF AN EBT REACTOR . . . . . . . . . . 8

4. DISCUSSION .................... 18

REFERENCES .......................... 20 


\section{THIS PAGE}

\section{WAS INTENTIONALLY LEFT BLANK}




\section{ABSTRACT}

The heating of an ELMO Bumpy Torus (EBT) reactor plasma by microwaves (ECH) and neutral beams is discussed. Although past and present EBT experiments use ECH for formation of the hot electron rings (annuli) and for heating of the bulk (toroidal) plasma, an EBT reactor and future proposed experiments may use neutral beams as well as ECH, or a combination of both, for heating of the toroidal plasma. When plasma is heated to ignition in a reactor, the bulk heating is turned off, but the ring heating must be sustained throughout the steady-state operation. For microwave heating in a large, high density reactor plasma, considered are questions of microwave energy penetration and accessibility (to both the ordinary and extraordinary modes). Microwave frequencies required are $120 \mathrm{GHz}$ for the toroidal plasma heating and $\sim 60-70 \mathrm{GHz}$ for the ring formation. These requirements are similar to those frequencies for the proposed EBT-II experiment. Because energetic particles having large pitch angle are well confined in EBT, perpendicular injection of neutral beams appears very attractive. The possibility of perpendicular injection, coupled with the large aspect ratio and relatively small plasma minor radius $(\sim 1 \mathrm{~m})$ for an EBT reactor, means that neutral beams of the type being developed for the Tokamak Fusion Test Reactor (TFTR) ( $150 \mathrm{keV})$ will be sufficient. 


\section{INTRODUCTION}

In the present EBT devicel all heating is provided by microwaves through the electron cyclotron resonance interaction. In addition, the microwaves play an essential role by producing the high beta hot electron annuli which provide macroscopic stability for the toroidal plasma component. The electron cyclotron heating $(\mathrm{ECH})$ is a comparatively well understood technique, which has been demonstrated successfully in EBT-I, 1,2 tokamaks, ${ }^{3}$ and other devices. Because no nonlinear coupling processes are involved, there are no electric field thresholds which must be exceeded, and therefore excessive microwave field intensities are not required. As a result, the extrapolation to larger, higher density EBT devices does not necessitate the exploitation of qualitative new heating mechanisms, but only involves scaling of currently used linear cyclotron absorption. The important questions to be addressed in scaling to a reactor regime are

1) Can the microwave energy in a large, high density device penetrate through the regions of evanescence to the cyclotron resonance layer?; and

2) If the resonance surfaces are accessible, can the microwave energy be directed to the plasma core of the device and not be simply deposited on the plasma surface?

In Sect. 2 is given a brief description of the ECH process, particularly as it relates to EBT. The heating mechanism is described and questions of accessibility are discussed. Calculations of ordinary-mode damping rates and ray path plots are presented for reactor plasma conditions.

Although the primary heating in EBT is provided by ECH, other techniques, such as neutral beam heating, may also be used successfully to heat the toroidal plasma component. Neutral beam heating may be employed as supplementary heating to reduce microwave heating requirements, as well as to provide flexibility in tailoring profiles for optimum stability and confinement. Neutral beam injection heating will have a major part in the EBT-II experiment. ${ }^{4}$ This experiment will provide valuable data and experience applicable toward an EBT reactor. ${ }^{5}$

Because of the large aspect ratio and relatively small minor radius ( $\sim \mathrm{m}$ ) in the EBT reactor, neutral beams. of moderate energy ( $150 \mathrm{keV}$ ) 
will be sufficient to heat an EBT reactor. In addition in EBT, particles with large perpendicular energies (large pitch angle) are well confined. Therefore, in contrast to tokamaks, perpendicular injection of neutral beams may be possible for an EBT reactor. In Sect. 3, some aspects of the application of neutral beams to an EBT reactor are discussed.

\section{MICROWAVE HEATING OF AN EBT REACTOR}

Electron cyclotron heating $(\mathrm{ECH})$ is a linear resonance process which has been successfully applied in a number of devices. For a review, particularly as applied to EBT-I, see Dandl, Eason, and Ikegami. ${ }^{2}$ The basic resonance can be obtained from consideration of electromagnetic waves in a simple cold magnetized plasma. For right-circularly polarized waves (extraordinary mode), propagating along the magnetic field, the refractivity index $n(\omega)$ satisfies the dispersion relation

$$
n^{2}(\omega)=\frac{c^{2} k^{2}}{\omega^{2}}=1-\frac{\omega_{p e}^{2}}{\omega\left(\omega-\Omega_{e}\right)},
$$

where

$$
\begin{aligned}
\omega_{p e} & =\text { electron plasma frequency } \\
\Omega_{e} & =|e| B / m_{e} c=\text { electron cyclotron frequency } \\
\omega & =\text { wave frequency. }
\end{aligned}
$$

At a location in the plasma where $\Omega_{e}=\omega$, the refractive index is infinite, and the waves are absorbed. For a low density cold plasma, the rate of energy absorption can be determined from the motion of single particles in the microwave fields. ${ }^{6}, 7$ A particle in an inhomogeneous magnetic field, $\Omega_{e}(z) \simeq \omega+\Omega^{\prime} z$, passing through the resonance zone, $\Omega_{e}=\omega$, will suffer a change in perpendicular energy, $\delta W_{\perp}$, due to acceleration by the right-circularly rotating component of the electric field, $E_{\perp}$. This change in energy can be either positive or negative depending upon the gyrophase angle of the particle relative to the wave field. However, when averaged over a random phase angle, the energy gain is positive and proportional to the wave energy density, $E_{\perp}^{2}$, and the length of time spent in the resonant region, $\Delta t=1 / \Omega^{-} v_{\|}$, 
ORNL/DWG/FED $78 \cdot 279$

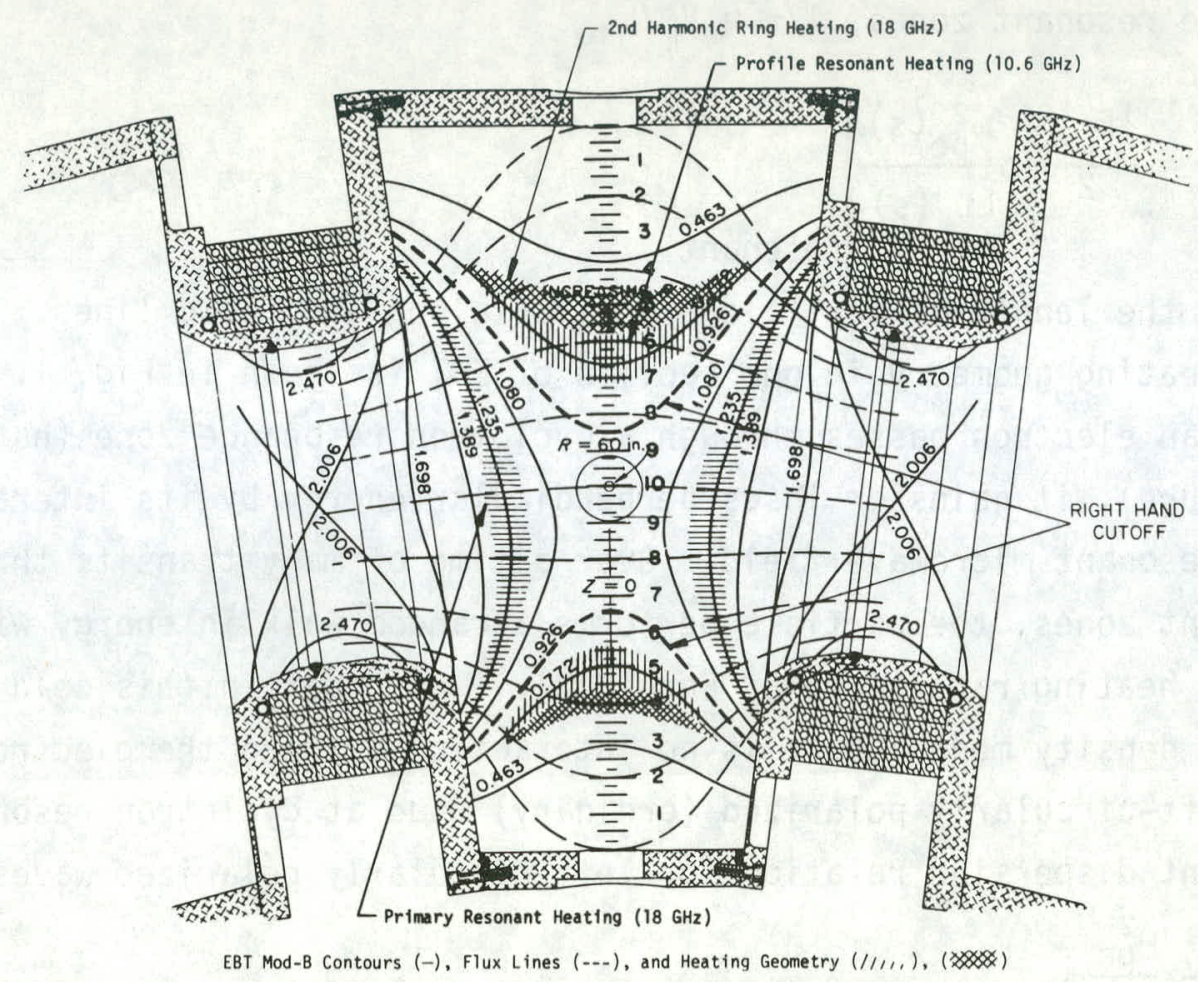

Fig. 1. Configuration of magnetic field in the ELMO Bumpy Torus. Two microwave frequencies $(10.6 \mathrm{GHz}$ and $18 \mathrm{GHz}$ ) are fed from the opening on the inside wall of the cavity. The wave mode at the injection port is ordinary rather than extraordinary. The hot electron ring (runaway electrons) is observed to be a narrow belt encircling the local second harmonic resonance (for $18 \mathrm{GHz}$ ) region in the midplane. The maximum microwave heating takes place at the cyclotron resonance zone (hatched in the figure). 


$$
\left\langle\delta W_{\perp}\right\rangle=\frac{\pi e^{2}\left|E_{\perp}\right|^{2}}{2 m \Omega \cdot V_{\|}}
$$

The net heating rate is obtained by multiplying this average energy increment by the particle density and the rate at which particles pass through the resonant zones, $1 / \tau=V_{\|} / L$,

$\frac{d}{d t}\left\langle\delta W_{\perp}\right\rangle=\left.\frac{\left|E_{\perp}\right|^{2}}{2} \frac{\omega_{p e}^{2}(s)}{L \Omega^{\prime}(s)}\right|_{\text {resonant }}$

where $L$ is the length of particle excursion along the field lines.

The heating geometry in one section of EBT is shown in Fig. 1. Each time an electron passes through a cyclotron resonance zone (hatched in the figure), it gains or loses perpendicular energy by its interaction with the resonant microwave field. Over a time of many transits through the resonant zones, the particle performs a random walk in energy with an average heating rate given by Eq. (2.3). Note that in this cold plasma low density model there is no interaction between the electrons and the left-circularly polarized (ordinary) mode at cyclotron resonance. The relevant dispersion relation for left-circularly polarized waves is $n^{2}(\omega)=1-\frac{\omega_{\text {pe }}^{2}}{\omega^{2}}$,

which has no resonances at $\omega=\Omega_{e}$.

The effects of finite temperature, finite wavelength, and arbitrary angle of propagation can be included by means of a quasi-linear treatment of the Vlasov equation with a specified microwave field distribution. ${ }^{7,8}$ A more straightforward approach, however, is to consider the linear damping of waves in a finite-temperature, uniform, magnetized plasma. ${ }^{9}$ Two basic mechanisms of resonant interaction are involved in the cyclotron absorption of electromagnetic waves by a finite-temperature plasma. One of these is associated with the presence in the wave of a circularly polarized component of the electric field rotating in the same direction as the Larmor rotation of an electron in the magnetic field. If the Doppler condition is fulfilled, $\omega=\Omega_{e}+k_{\|} V_{\|}$, this component is in a fixed phase with respect to the electron transverse velocity, thereby 
ensuring a resonant energy exchange between wave and particle. This mechanism is responsible for the damping of extraordinary waves propagating nearly along the magnetic field. The heating produced goes predominantly into perpendicular energy, and the efficiency of this mechanism decreases as the angle of propagation increases relative to the magnetic field.

If the wave vector has a component $k_{\perp}$ perpendicular to the magnetic field, there is a resonant interaction of the particle with the component of the microwave electric field parallel to $\underline{B}$. If the electron gyroradius is finite and the Doppler condition is satisfied, this force is nonzero and constant in time. This mechanism accounts for the damping of the ordinary wave propagating nearly perpendicular to $\underline{B}$. The heating goes predominantly into parallel energy, and the efficiency decreases as the angle of propagation relative to $\underline{B}$ decreases.

The combined effect of the above mechanisms affords a qualitative explanation of the angular dependences of the cyclotron absorption rates. Neglecting the effects of ray focusing, the wave energy flux, $S$, along a ray path, $s$, is given by

$\frac{\mathrm{dS}}{\mathrm{ds}}=-\alpha \mathrm{S}$,

where $\alpha=2 k_{j}$ is the energy absorption coefficient, and $k_{i}$ is the imaginary part of the wave number. Figures $2 a$ and $b$ show the energy absorption coefficient $\alpha$ in $\mathrm{cm}^{-1}$ at exact resonance for ordinary and extraordinary modes as a function of angle of propagation, $\theta$, for various plasma densities, $q=\omega_{p e}^{2} / \omega^{2}$. The temperature $(\sim 12 \mathrm{keV})$ and wave frequency $(120 \mathrm{GHz})$ were chosen to be representative of an EBT reactor. The angular dependence discussed above can clearly be seen in the figures.

Another important feature is that the absorption rate increases with density for the ordinary mode but decreases for the extraordinary mode. In both cases, the absorption rates are very large. Even for the ordinary mode the damping length, $L=1 / \alpha$, is less than $1 \mathrm{~cm}$ for $\theta \geqslant 60^{\circ}$ and $\omega_{\mathrm{pe}}^{2} / \omega^{2} \geq 0.5$. It should be noted that damping of the ordinary mode is a finite Larmor-radius effect, whose strength is proportional to $k_{1} \rho_{e}$. For a relatively low temperature $(2200 \mathrm{eV})$, low density $\left(210^{12} \mathrm{~cm}^{-3}\right)$ 

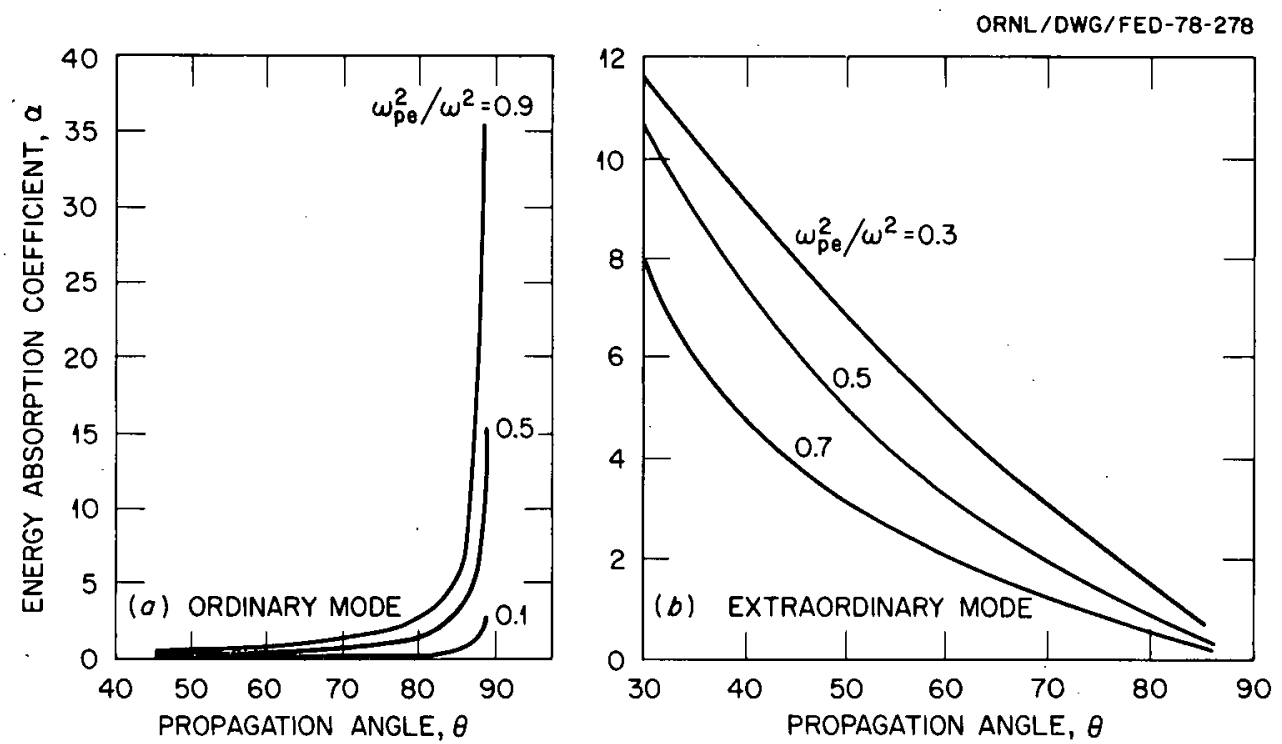

Fig. 2. Energy absorption coefficient (damping rate) a versus propagation angle $\theta$ for various values of $\omega_{\text {pe }}^{2} / \omega^{2}$ for $(a)$ ordinary and
(b) extraordinary modes. 
plasma, such as the toroidal core plasma of EBT-I, ordinary-mode absorption is a very weak process. However, for a hot $\left(T_{e}>2 \mathrm{keV}\right)$, dense $\left(n_{e} \geq 10^{13}\right)$ plasma, such as projected for EBT-II or EBTR, the ordinary mode is very heavily damped.

Finite Larmor-radius effect also permits resonant wave-particle interactions at harmonics of the cyclotron frequency, $\omega=n \Omega_{e}+k_{\|} V_{\|}$. The strength of these interactions is proportional to $\left(k_{\perp} \rho_{e}\right)^{n}$ and decreases rapidly at higher harmonics. However, the second harmonic resonance is important and permits strong perpendicular heating at $\omega \simeq 2 \Omega_{\mathrm{e}}$, particularly by the extraordinary mode. It is the second harmonic resonance which seems to produce the perpendicular relativistic electron component forming the high-beta annulus (cross-hatched in Fig. 1).

Another important consideration in extrapolating $\mathrm{ECH}$ to the reactor regime is the accessibility of the resonant zone. It can be seen from Eq. (2.1) that for values of $\Omega_{e}$ such that

$\Omega_{e}<\omega<\omega_{R}=\frac{1}{2}\left(\Omega_{e}+\sqrt{\omega_{\text {pe }}^{2}+\Omega^{2}}\right)$,

the refractive index is imaginary $\left[n^{2}(\omega)<0\right]$, and the wave is evanescent. The implication is that a wave propagating from the low field side of the cyclotron resonance $\left(\Omega_{e}<\omega\right)$ will reach a point at which $\omega=\omega_{R}$ will be reflected and will never reach the resonant region. A point at which $\omega=\omega_{R}$ is called a right-hand cutoff since the refractive index $n^{2}(\omega)$ for the right-circularly polarized wave vanishes at such a point. The location of the right-hand cutoff is essentially independent of temperature or angle of propagation except for very low density plasmas. The importance of this to EBT stems from the fact that microwaves propagating from near the midplane of a mirror sector must, in general, cross a right-hand cutoff layer to reach the cyclotron resonance layer. The location of the right-hand cutoff zone is shown in Fig. 1 (heavy dashed lines) for a model density profile. For the relatively high density chosen, almost the entire core of the plasma is cut off from the extraordinary mode. Of course, if the central density remains below the critical density, the entire plasma is accessible to the ordinary mode, 
and heating of the bulk plasma can be accomplished by this means.

Furthermore, the second harmonic resonant zone will be accessible to both the ordinary and extraordinary modes, provided $\omega_{\text {pe }}<\omega / \sqrt{2}$ at the second harmonic resonance. Therefore, we can expect to maintain an annulus for any achievable density.

Finally, we consider whether microwave energy can be propagated into the center of a hot, high density plasma without being deposited near the surface. Fiqure 3 shows results from a three-dimensional (3-D) ray tracing code in which ordinary-mode rays were injected at varying angles into a mirror equilibrium. The parameters chosen were $f_{\mu}=120$ $\mathrm{GHz}, \mathrm{n}_{\mathrm{e}}=1.5 \times 10^{14} \mathrm{~cm}^{-3}$, and $\mathrm{T}_{\mathrm{e}}=12 \mathrm{keV}$. The injection point was located at the nidplane just outside the plasma, $r=100 \mathrm{~cm}$. Also shown in Fig. 3 are the magnetic field lines and cross sections of the cyclotron resonant surface and right-hand cutoff surface. The density profile was modeled to be constant along magnetic field lines and to decrease radially along the $z$ axis (magnetic axis). For the ordinary mode, the rays are nearly straight but with slight curvature due to density gradients. Each asterisk marks a 10\% decrement in wave power. It can be seen that little power is absorbed at the surface, while all of the power is absorbed from those rays which pass through the resonant zone near the center of the plasma.

\section{NEUTRAL BEAM HEATING OF AN EBT REACTOR}

In EBT, energetic particles having large pitch angle are well confined. This makes perpendicular injection practical. The possibility of perpendicular injection, coupled with the large aspect ratio and relatively small reactor plasma minor radius $(\sim 1 \mathrm{~m})$, means that neutral beams of TFTR type ( $150 \mathrm{keV}$ ) will be sufficient.

Injection of a neutral beam at an angle far from perpendicular to the magnetic field would require modification of several of the blanketshield-magnet sectors (modules). In addition to the inefficiency (due to long absorption length), engineering difficulty, and large extrapolation in neutral beam technology, such near-parallel injection is not attractive for efficient plasma heating since some of the beam particles 


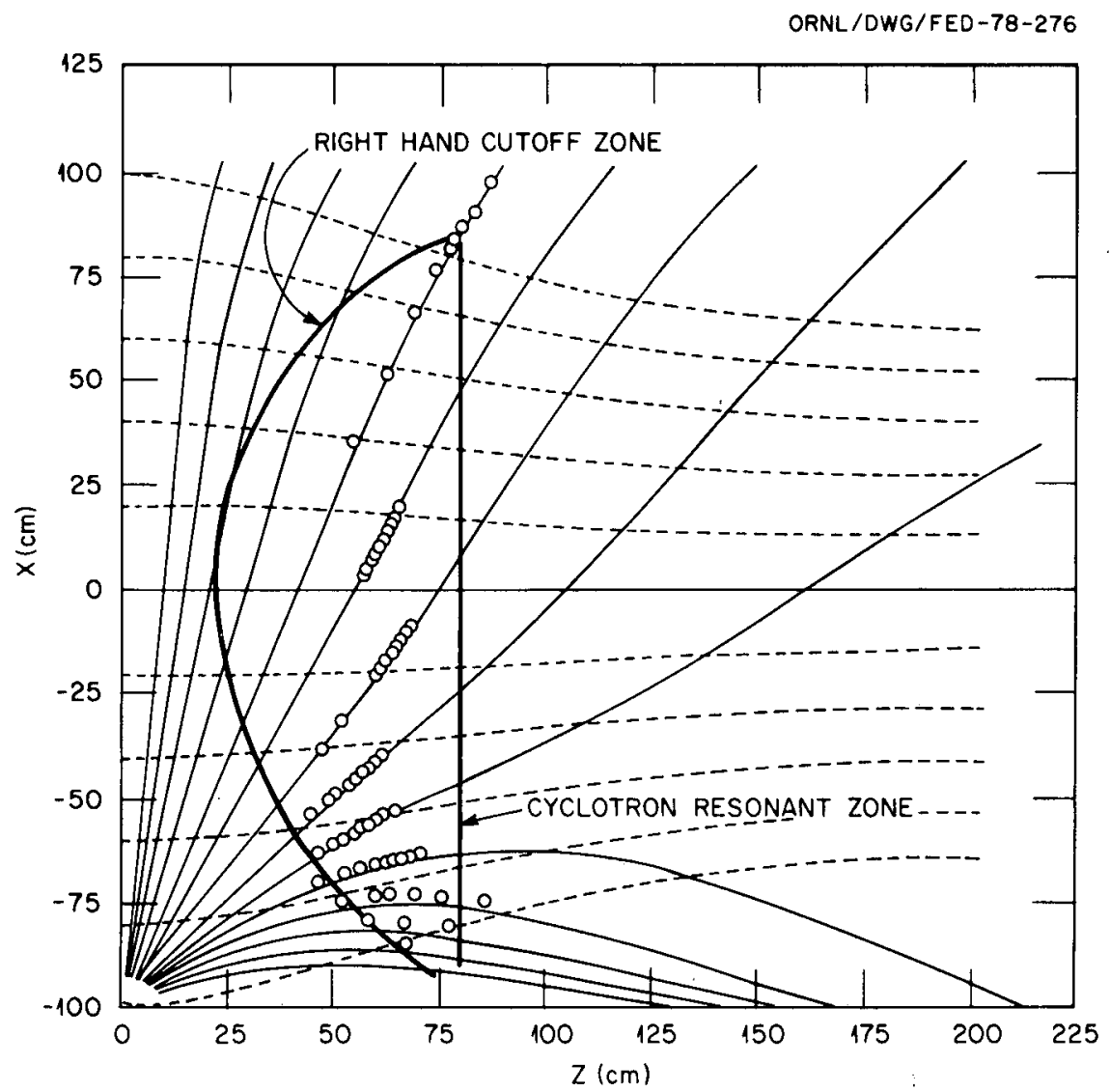

Fig. 3. Ordinary-mode propagation. 
would be ionized on open-drift surfaces. The slowing down time for the resulting hot ions is much longer than the time required for the hot ion to drift to the wall.

Perpendicular or near-perpendicular injection would entail only minor modifications to the structure of the device. The large aspect ratio of the machine provides excellent access from either the outside or the top. Calculations that follow will consider perpendicular injection. Figure 4 shows the geometry for radial or vertical injection. Pencil beams which give the most optimistic deposition result have been considered.

The rate at which the beam particles are ionized along the beam path can be obtained in conventional calculations. ${ }^{10}$ The number density of beam particles $N_{B}$ as a function of distance along the beam path $s$ satisfies

$N_{B}(s)=N_{B}(-\infty) e^{-D(s)}$,

where $\lambda(s)$ is the mean free path of a neutral beam particle at point $s$, and the absorption decrement $D(s)$ is given by

$D(s)=\int_{-\infty}^{s} \frac{d s^{\prime}}{\lambda\left(s^{\prime}\right)}=\int_{-\infty}^{s} \frac{n\left(s^{\prime}\right)}{\lambda_{0} n_{0}} d s^{\prime}$,

where $\lambda_{0}$ is the mean free path at maximum density $n_{0}$. Recent calculations indicate a nearly constant density within the plasma core for EBT-I.l The extremes of either a constant profile or a parabolic one have been considered here. In general, for a model density profile of the form $n(r)=n_{0}\left[1-\left(\frac{r^{2}}{a^{2}}\right)^{p}\right]$,

the absorption decrement $D(s)$ can be evaluated directly,

$$
D(s)=\frac{1}{\lambda_{0}} \times \begin{cases}0 & , s<-a . \\ s+a-\frac{a}{2 p+1}\left[\left(\frac{s}{a}\right)^{2 p+1}+1\right] & ,-a<<a . \\ \frac{4 p}{2 p+1} a & , s>a .\end{cases}
$$


ORNL-OWG 76-14068

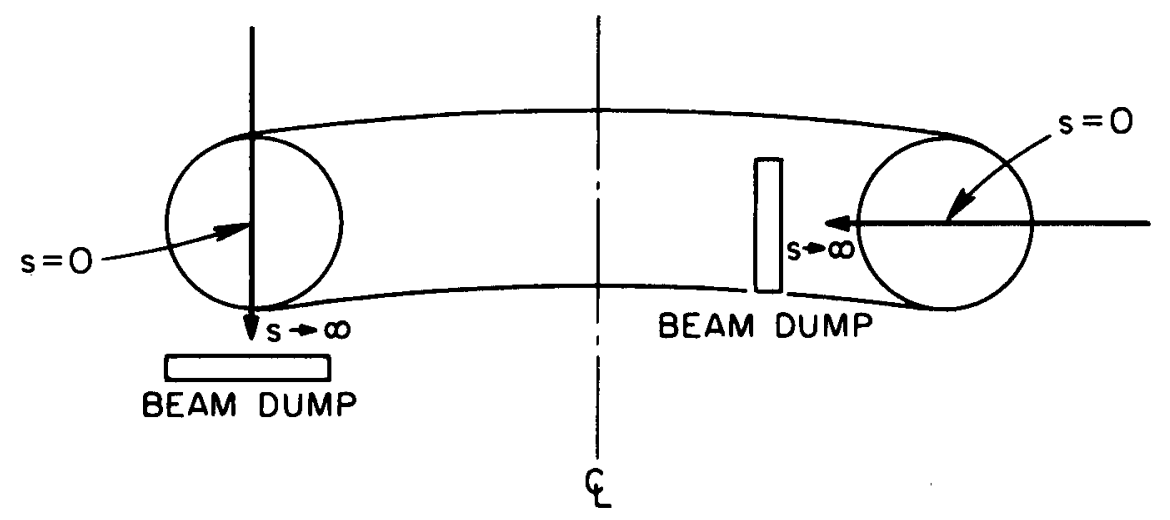

Fig. 4. Side view of torus showing vertical and horizontal injection. 
The fraction of beam particles not absorbed in passing through the plasma column, $N_{B}(\infty) / N_{B}(-\infty)$, is given by

$N_{B}(\infty) / N_{B}(-\infty)=\exp \left(-\frac{4 p}{2 p+1} \frac{\partial}{\lambda_{0}}\right)$.

In order to absorb at least $80 \%$ of the beam particles, it is necessary to have $a / \lambda_{0} \geq 0.8+0.4 / p$. For a parabolic profile $(p=1)$, one must have $a / \lambda_{0} \geq 1.2$; for a constant or sharp boundary profile $(p \rightarrow \infty)$, it is necessary that $a / \lambda_{0} \geq 0.8$.

The mean free path for absorption of an energetic neutral is approximated by

$\lambda_{0}^{-1}=\left[n_{0} \sigma_{C X}\left(V_{B}\right)+\sigma_{i}\left(V_{B}\right)\right]$.

Figure 5 shows $\lambda_{0}$ for various beam energies and plasma densities. This calculation is based on published data for the charge exchange $\sigma_{c x}$ and proton ionization $\sigma_{i}$ cross section. ${ }^{11}$ Electron impact ionization is negligible at large beam particle velocities $V_{B}$, and alpha particle and impurity contributions to ionization are assumed to be small. For an EBT reference reactor, 5 taking a plasma radius of $a=1.0 \mathrm{~m}$ and a peak plasma density of $n_{0}=2 \times 10^{20} \mathrm{~m}^{-3}$, it can be seen from Fig. 5 that $a / \lambda_{0} \simeq 3$ for $200-k e V$ deuteron beam. This leads to about $98 \%$ absorption of the beam for the parabolic density profile case and about $>99 \%$ absorption for the sharp boundary profile. For a 150-keV beam, $a / \lambda_{0} \simeq 4$, which leads to $\sim 100 \%$ absorption.

After the initial ionization of beam particles, the fast ions tend to spread uniformly over a toroidal drift surface before their energy diffuses radially, because of the ordering of the drift (poloidal), bounce, and collision times for energetic ions. A typical ordering for energetic ions is as follows

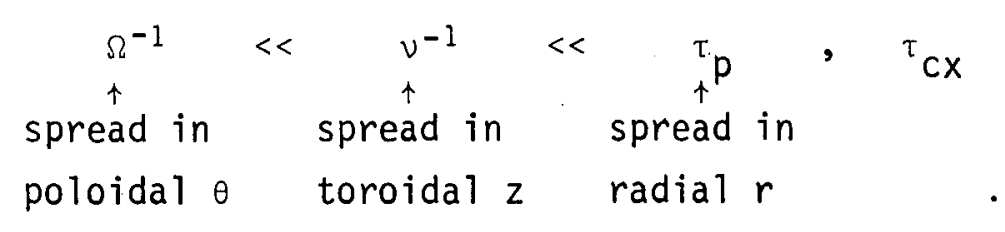




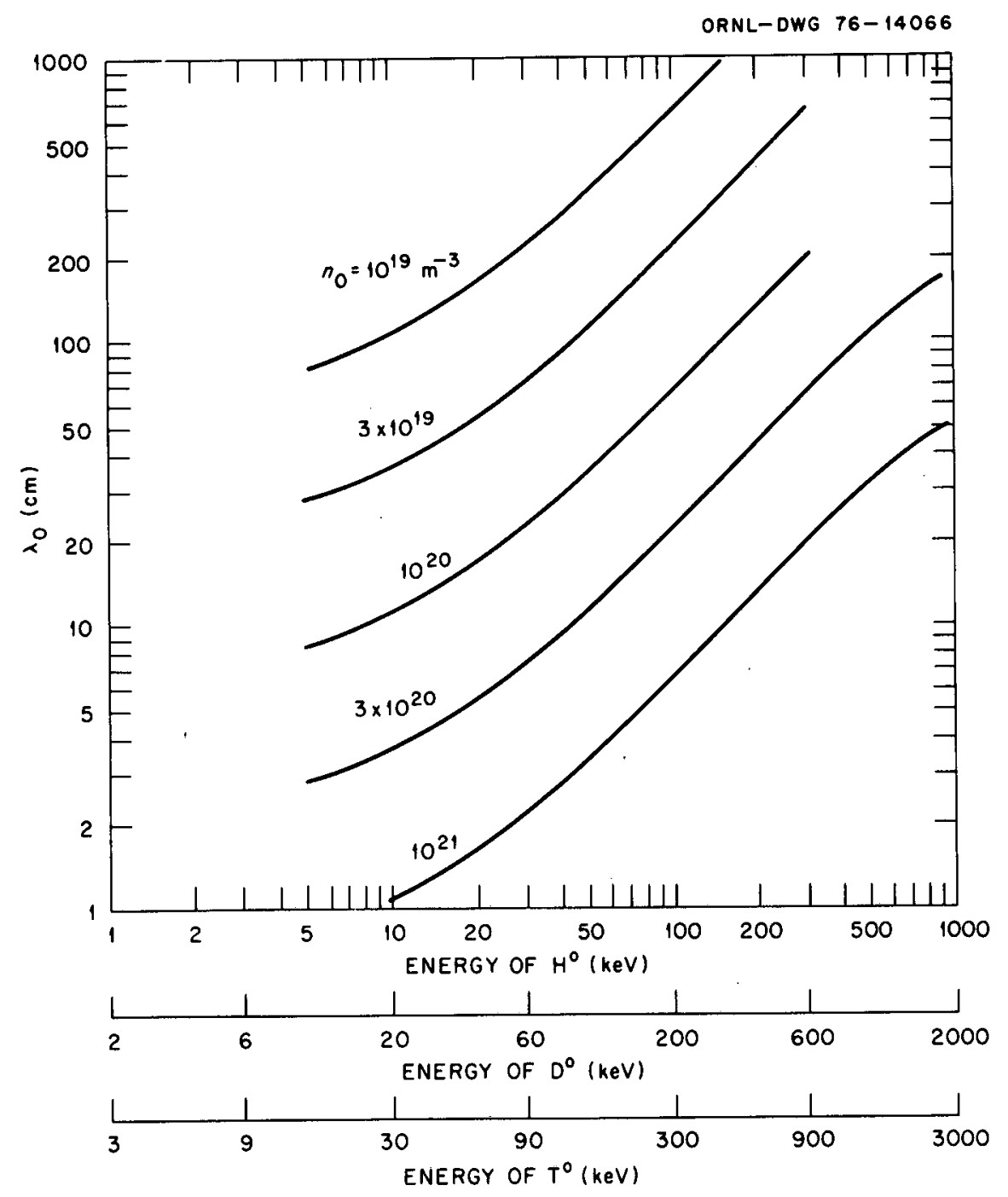

Fig. 5. Absorption mean free path versus energy of atomic hydrogen, deuterium, and tritium neutral beams including impact ionization and charge exchange for injection into D-T plasma. 
That is, the poloidal drift period and ninety-degree scattering time are much less than the time required to move radially through the plasma. Assuming a thin beam (pencil beam), the fast particle rate is

$\dot{n}_{f}=\sum_{ \pm s}-\frac{d N_{B}(s)}{d s} \frac{d s}{d r} \frac{1}{4 \pi^{2} R_{0} r}$,

where the area of the poloidal drift surface at midplane radius $r$ is approximated by $4 \pi^{2} R_{0} r$ with $R_{0}$ being the toroidal major radius. The rate at which fast ions are produced within a simple toroidal volume of minor radius $r$ is given by

$$
\begin{aligned}
\dot{N}(r) & =\int_{0}^{r} d r 4 \pi^{2} r{ }_{0} \dot{n}_{f}(r) \\
& =\frac{I_{B}}{e} \int_{0}^{r / a} d x H(x) .
\end{aligned}
$$

Here $I_{B}$ is the neutral beam current (in equivalent amperes, i.e., the ratio of beam power, $P_{B}$, in watts to beam particle energy, $E_{B}$, in electron volts) and the radial heat deposition profile $H(x)$

$H(x)=2\left(\frac{a}{\lambda_{0}}\right)\left(1-x^{2 p}\right) e^{-\frac{2 p}{2 p+1}\left(\frac{a}{\lambda_{0}}\right)} \cosh \left[\frac{a}{\lambda_{0}} x\left(1-\frac{x^{2 p}}{2 p+1}\right)\right]$

is plotted in Fig. 6 for several values of $a / \lambda_{0}$ for the limits of $p=1$ and $p \rightarrow \infty$, parabolic and constant density profiles, respectively. The area under $H(x)=H(r / a)$ is the fraction of the injected neutral beam which is converted to fast ions within a given toroidal volume. For perpendicular injection the energetic ions are well confined; this also corresponds to the heating profile (except for energy lost by escaping neutrals).

The partitioning of the energy between the background plasma electrons and ions (including charge exchange losses) is given by the functions $G_{e}$ and $G_{j}$ derived by Callen et al., ${ }^{12}$ and plotted in Fig. 7. The approximate fit to the $G_{e}$ and $G_{j}$ curves given in Ref. 12 yields ${ }^{13}$ 


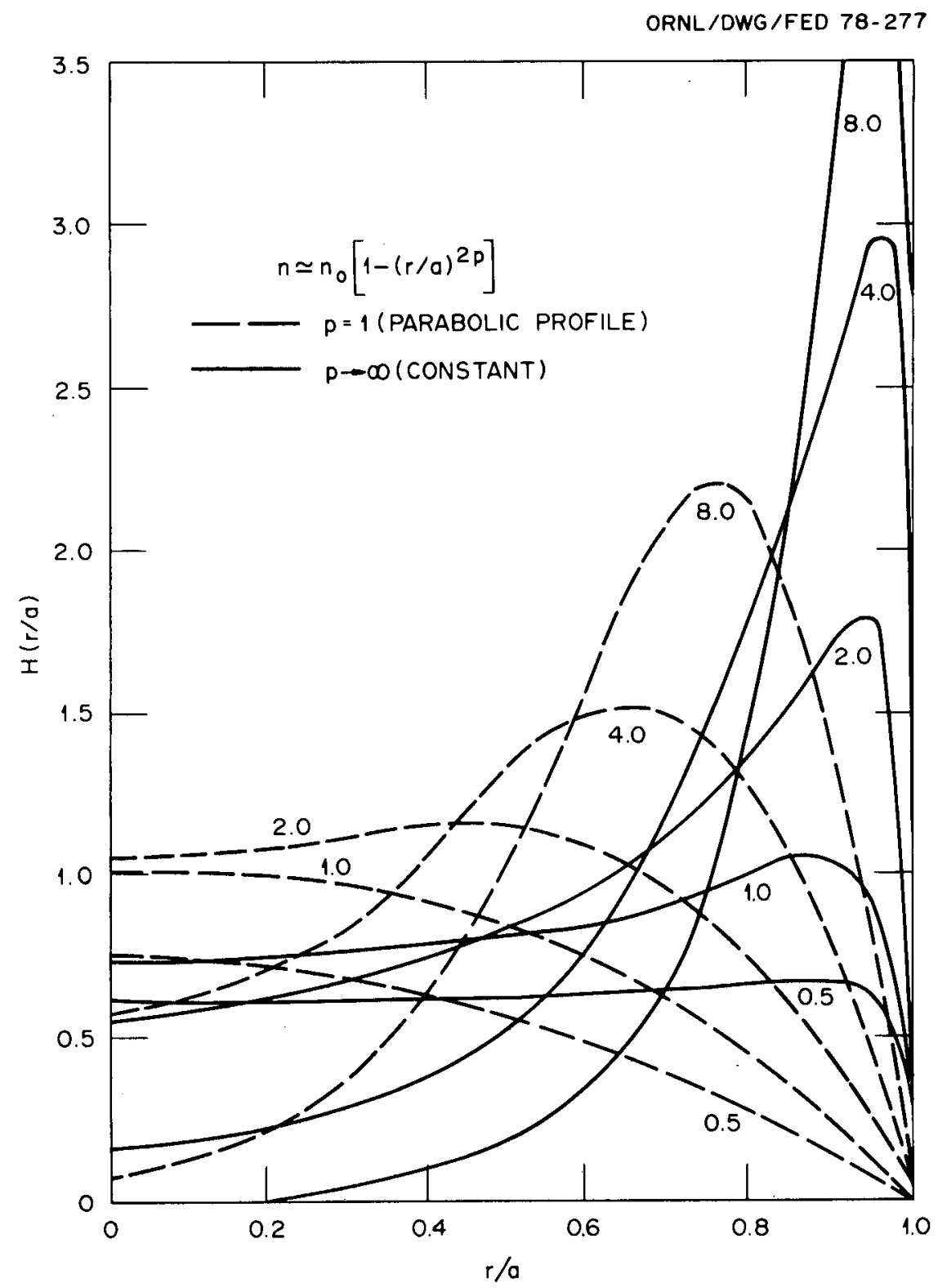

Fig. 6. Fast ion deposition $H(r / a)$. Curves are labelled with $a / \lambda_{0}$. 


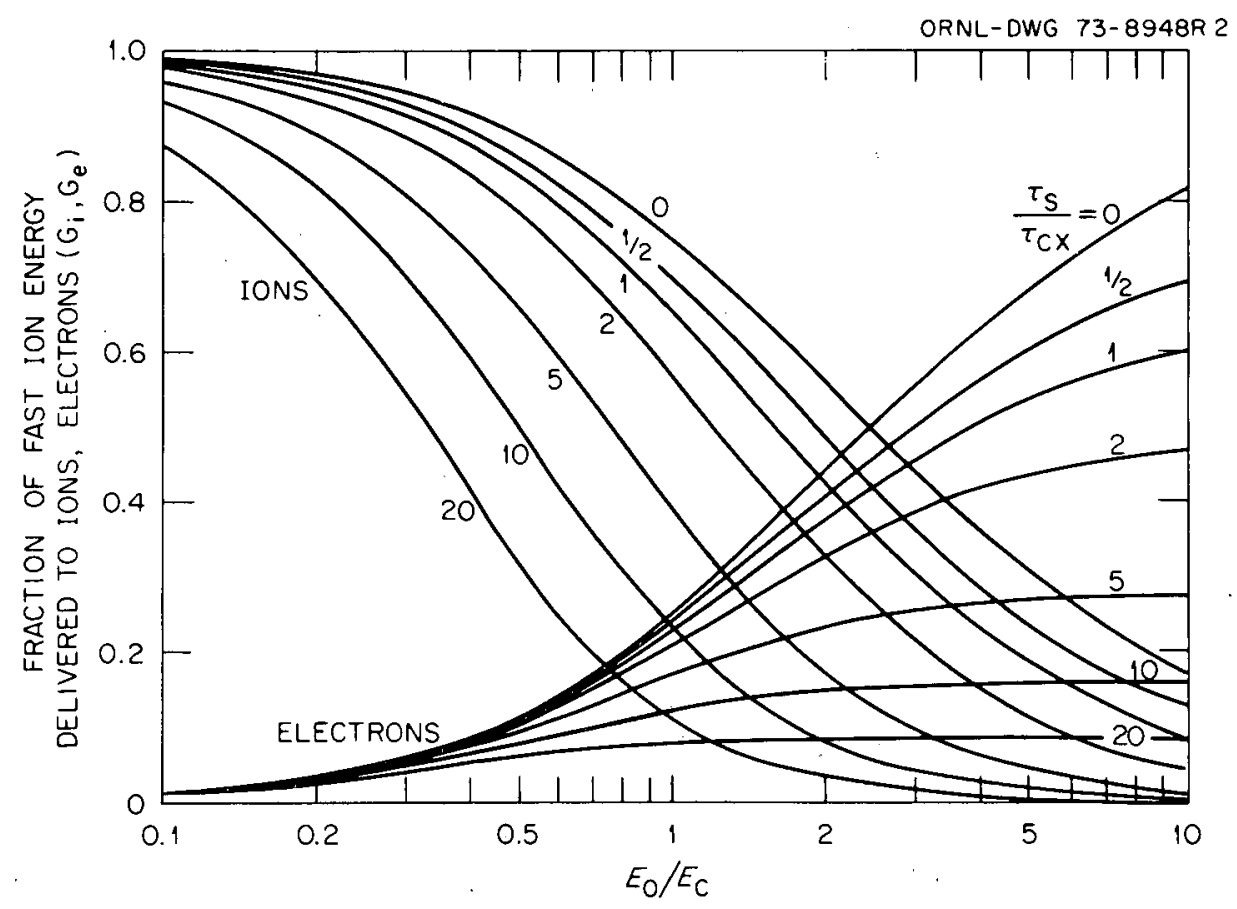

Fig. 7. Ion and electron energy transfer functions $\left(G_{j}, G_{e}\right)$ for various $\tau_{s} / \tau_{c x}$. 


$$
\begin{aligned}
& G_{i} \simeq 0.5 \exp \left[-0.1 \frac{E_{B}}{E_{c r}}\left(1+0.5 \frac{\tau_{s}}{\tau_{c x}}\right)\right] \\
&+0.5 \exp \left[-0.6 \frac{E_{B}}{E_{c r}}\left(1+0.5 \frac{\tau_{s}}{\tau_{c x}}\right)\right], \\
& G_{e}=1-G_{i}-G_{c x},
\end{aligned}
$$

where

$$
G_{C X} \approx\left\{\begin{array}{l}
1-\exp \left[-0.12 \frac{E_{B}}{E_{c r}}\left(\frac{\tau_{s}}{\tau c x}\right)\right], \frac{E_{B}}{E_{C r}} \leq 1 . \\
1-\exp \left[-0.12\left(\frac{E_{B}}{E_{c r}}\right)^{1 / 2}\left(\frac{{ }^{\tau} s}{\tau_{c X}}\right)\right], \frac{E_{B}}{E_{C r}}>1 .
\end{array}\right.
$$

$E_{c r}$ is the critical energy, i.e., the critical energy at which beam energy is transferred equaliy to the electrons and ions at equal rate $\left(E_{c r} \sim 14.8 T_{e}\right)$; $\tau_{s}$ is the spitzer ion-electron momentum exchange time; and $\tau_{c x}$ is the time for charge exchange.

The beam heating term in the EBT plasma simulation model (point model) 13 is approximated by

$P_{B}=\frac{E_{B} \dot{N}(a) F}{2 \pi^{2} a^{2} R_{0}}$.

The factor $F$ accounts for the rapid escape of energetic ions through any velocity space loss cones. The beam particle source term in the model $1^{13}$ is

$S_{B}=\frac{\dot{N}(a) F}{2 \pi^{2} a^{2} R_{0}} \frac{\sigma_{i}}{\sigma_{i}+\sigma_{c x}}$,

where it is noted that charge exchange does not change the total number of ions, and

$\dot{N}(a)=\frac{I_{B}}{e} \int_{0}^{1} d x H(x)=\frac{P_{B}}{e E_{B}}\left[1-\frac{N(\infty)}{N(-\infty)}\right]$.

A variety of effects can reduce the containment factor $F$ below unity. One must obviously avoid having magnetic field lines intersect the plasma 
chamber's first wal1. To lowest order, the field line shape in EBT is sinusoidal with the midplane radius bulging $\sqrt{M}$ times the mirror throat radius, where $M$ is the mirror ratio. From equilibrium and maximum use of the magnetic field for containing plasma in an EBT reactor and to properly handle this field line geometry, the displaced aperture ogee first-wall shape has been adopted in the reference design. ${ }^{5}$ The design accounts for the relatively large gyroradii of the energetic ions which have a 20-cm-thick cold plasma zone. One of the concerns is the complicated banana and superbanana drift orbits of the particle guiding center. Mirror and toroidal curvature, finite beta, and radial electric fields make general statements difficult, but the encouraging observation is that very large aspect ratios (desirable also from the standpoint of maintenance ease) tend to have fairly well-centered drift surfaces. The fraction of the chamber cross section which has ideal confinement has been studied under a variety of conditions within the EBT Theory Program. ${ }^{1,4}$

\section{DISCUSSION}

In EBT there are two types of heating required:

1) Heating of some electrons to relativistic and near relativistic energies to form the hot electron rings (annuli) needed for MHD stabilization of the bulk (toroidal) plasma, and

2) Heating of the bulk plasma. In a reactor when plasma is heated to ignition, the bulk heating is turned off, but the ring heating must be sustained throughout operation. Past and present experiments use ECH to heat the bulk plasma as well as the ring electrons; however, an EBT reactor and future proposed experiments may use neutral beams as well as $\mathrm{ECH}$, or a combination of both, to heat the toroidal plasma. It should be noted that a pure beam heated plasma might have different thermal stability properties than a plasma whose ions are heated by electron-ion equilibration. Theoretical studies are under way to determine the properties of beam heated EBT plasmas. The proposed EBT-II experiment ${ }^{4}$ will also provide valuable information in this area.

Heating of an EBT reactor plasma with microwaves (ECH) is discussed in Sect. 2. The questions of microwave energy penetration and 
accessibility in a large high density reactor plasma are addressed. Microwave frequencies required are $120 \mathrm{GHz}$ for the toroidal plasma heating and $\sim 60-70 \mathrm{GHz}$ for the ring formation. These frequencies are similar to the requirements of the proposed EBT-II experiment. ${ }^{4}$ On the basis of our calculations to date for reactor plasmas, the findings are:

- The second harmonic resonant zone (the location at which the annulus should form) should be accessible to both the ordinary and extraordinary modes.

- For densities less than the critical density $\left(\omega_{\mathrm{pe}}<\omega_{\mu}\right)$, the entire plasma should be accessible to the ordinary mode.

- At reactor temperatures and densities the ordinary mode is strongly absorbed at the cyclotron resonance surface.

- Ordinary-mode energy can be deposited in the plasma center.

Neutral beam injection heating of an EBT reactor plasma is discussed in Sect. 3. Neutral beam heating is the most technically advanced and experimentally proven form of auxiliary heating for tokamaks and 2XII-B mirror. It is also one of the prime contenders for bulk heating of an EBT plasma. As the plasmas get larger and denser (characteristic of reactor plasmas), beam penetration becomes the major problem. Injection efficiency drops sharply beyond 200-keV deuteron energy for positive ion sources. Above $200 \mathrm{keV}$, negative ion sources permit a much higher neutral production efficiency, and one can overcome the penetration problem at these energies. However, negative ion source technology is in its early development stage as is the high power high frequency microwave development program. In EBT, particles with large perpendicular energies are well confined. This makes perpendicular injection practical and possible. Because of the large aspect ratio and relatively small plasma minor radius $(\sim 1 \mathrm{~m})$ coupled with the possibility of perpendicular injection, neutral beams of moderate energy (of TFTR type, $2150 \mathrm{keV}$ ) will be sufficient to heat an EBT reactor. 


\section{REFERENCES}

1. R. A. Dandl et a1., Proc. 5th Intl. Conf. on Plasma Physics and Controlled Nuclear Fusion Research, Vol. II, p. 141 (1975);

R. A. Dandl et a 1., Research Program for Plasma Confinement and Heating in ELMO Bumpy Torus Devices, Oak Ridge National Laboratory Report, ORNL/TM-4941 (June 1975); The ELMO Bumpy Torus Program, ORNL/TM-5451 (Apri1 1976).

2. R. A. Dand 1, H. O. Eason, and H. Ikegami, "Microwave Plasma Heating at the Electron Cyclotron Frequency, to be published in Nucl. Fusion.

3. V. E. Golant et al., Sov. Phys.-Tech. Phys. 17, 488 (1972); V. V. Alikaev et al., Plasma Physics and Controlled Nuclear Fusion Research, Nucl. Fusion Supplement 33 (1974).

4. R. A. Dandl et al., The EBT-II Conceptual Design Study, Oak Ridge National Laboratory, ORNL/TM-5955, to be published.

5. N. A. Uckan et a1., The ELMO Bumpy Torus (EBT) Reactor, Oak Ridge National Laboratory Report, ORNL/TM-6084, to be published.

6. A. F. Kukes, Plasma Physics 10, 367 (1968).

7. 0. C. Eldridge, Phys. Fluids 15, 676 (1972).

8. C. F. Kennel and F. Engelmann, Phys. Fluids 9,2377 (1966).

9. A. I. Akhiezer et a1., Plasma Electrodynamics, Vo1. I, Pergammon Press, 0xford (1976).

10. J. A. Rome, J. D. Callen, and J. F. Clarke, Nucl. Fusion 14, 141 (1975).

11. A. C. Riviere, Nucl. Fusion 11, 363 (1971).

12. J. D. Callen et a1., Proc. 5th Int1. Conf. on Plasma Physics and Controlled Nuclear Fusion Research, Vol. I, p. 645 (1975).

13. N. A. Uckan and C. L. Hedrick, Plasma Simulation and Modeling for ELMO Bumpy Torus Reactor, Oak Ridge National Laboratory Report, ORNL/TM-6019 (October 1977). 


\author{
ORNL/TM-6346 \\ Dist. Category UC-20a,d,g
}

INTERNAL DISTRIBUTION

1. L. A. Berry

2. J. D. Callen

3. R. A. Dand 1

4. R. A. Dory

5. G. G. Kelley

6. H. H. Haselton

7. P. N. Haubenreich

8. M. S. Lube11

9. 0. B. Morgan

10. H. Postma

11. M. W. Rosenthal

12. J. Sheffield
13. D. Steiner

14. D. B. Batchelor

15-39. N. A. Uckan

40-41. Laboratory Records Department

42. Laboratory Records, ORNL-RC

43. Document Reference Section

44-45. Central Research Library

46. Fusion Energy Division Library

47. Fusion Energy Division Communications Center

48. ORNL Patent Office

\title{
EXTERNAL DISTRIBUTION
}

49. Bibliothek, Max-Planck Institute für Plasmaphysik, 8046 Garching bei München, Federal Republic of Germany

50. Bibliothèque, Service du Confinement des Plasmas, C.E.A., B.P. No. 6, 92, Fontenay-aux Roses (Seine), France

51. Lung Cheung, Department of Electronics, University Science Center, The Chinese University of Hong Kong, Shatin,. N.T., Hong Kong

52. J. F. Clarke, Office of Fusion Energy, G-234, Department of Energy, Washington, DC 20545

53. D. Cohn, Massachusetts Institute of Technology, Cambridge, MA 02739

54. R. W. Conn, Fusion Technology Program, Nuclear Engineering Department, University of Wisconsin, Madison, WI 53706

55. CTR Library, c/o Alan F. Haught, United Technologies Research Laboratory, East Hartford, CT 06108

56. CTR Reading Room, c/o Allan N. Kaufman, Physics Department, University of California, Berkeley, CA 94720

57. Hatice Cullingford, Office of Fusion Energy, G-234, Department of Energy, Washington, DC 20545

58. J. Narl Davidson, School of Nuclear Engineering, Georgia Institute of Technology, Atlanta, GA 30332

59. Documentation S.I.G.N., Départment de la Fhysique du Plasma et de la Fusion Controlée, Association EURATOM-CEA sur la Fusion, Centre d'Études Nucléaires, B.P. 85, Centre du TRI, 38041 Grenoble, Cedex, France

60. W. R. Ellis, Office of Fusion Energy, G-234, Department of Energy, Washington, DC 20545

61. G. A. Emmert, Nuclear Engineering Department, University of Wisconsin, Madison, WI 53706 
62. Harold K. Forsen, Exxon Nuclear Co., Inc., 777 106th Avenue, N.E., C-000777, Bellevue, WA 98009

63. Harold P. Furth, Princeton Plasma Physics Laboratory, Princeton University, Forrestal Campus, P.0. Box 457, Princeton, NJ 08540

64. Roy W. Gould, California Institute. of Technology, Mail Stop 116-81, Pasadena, CA 91125

65. Charles R. Head, Office of Fusion Energy, G-234, Department of Energy, Washington, DC 20545

66. Robert L. Hirsch, Exxon Research and Engineering, P.0. Box 101, Florham Park, NJ 07932

67. Raymond A. Huse, Manager, Research and Development, Public Service Gas and Electric Company, 80 Park Place, Newark, NJ 07101

68. T. Hsu, Office of Fusion Energy, G-234, Department of Energy, Washington, DC 20545

69. V. E. Ivanov, Physical-Technical Institute of the Ukranian Academy of Sciences, Sukhumi, U.S.S.R.

70. D. L. Jassby, Princeton Plasma Physics Laboratory, P.0. Box 451 , Princeton, NJ 08540

71. A. Kadish, Office of Fusion Energy, G-234, Department of Energy, Washington, DC 20545

72. L. M. Kovrizhnikh, Lebedev Institute of Physics, Academy of Sciences of the U.S.S.R., Leninsky Prospect 53, Moscow, U.S.S.R.

73. Guy Laval, Groupe de Physique Théorique, Ecole Polytechnique, 91 Palaiseau, Paris, France

74. Library, Centre de Recherches en Physique des Plasma, 21 Avenue des Bains, 1007, Lausanne, Switzerland

75. Library, Culham Laboratory, United Kingdom Atomic Energy Authority, Abingdon, 0xon, 0x14 3DB, United Kingdom

76. Library, FOM-Institut voor Plasma - Fysica, Rijnhuizen, Jutphaas, Netherlands

77. Library, Institute for Plasma Physics, Nagoya University, Nagoya, Japan 464

78. Library, International Centre for Theoretical Physics, Trieste, Italy

79. Library, Laboratorio Gas Ionizzati, Frascati, Italy

80. Dsumber G. Lominadze, Academy of Sciences of the Georgian S.S.R., 8 Dzerzhinski St., 38004, Tbilisi, U.S.S.R.

81. Oscar P. Manley, Office of Fusion Energy, G-234, Department of Energy, Washington, DC 20545

82. D. G. McAlees, Exxon Nuclear Co., Inc., Research and Technology Laser Enrichment Department, 2955 George Washington Way, Richland, WA 99352

83. J. E. McCune, School of Engineering, Department of Aeronautics and Astronautics, B1dg. 37-391, Massachusetts Institute of Technology, Cambridge, MA 02139

84. Claude Mercier, Service du Theorie des Plasmas, Centre d'Études Nucleaires, Fontenay-aux-Roses (Seine), France

85. K. G. Moses, Office of Fusion Energy, G-234, Department of Energy, Washington, DC 20545 
86. Michael Murphy, Office of Fusion Energy, G-234, Department of Energy, Washington, DC 20545

87. D. Pfirsch, Institute for Plasma Physics, 8046 Garching bei München, Federal Republic of Germany

88. Plasma Physics Group, Department of Engineering Physics, Australian National University, P.0. Box 4, Canberra A.C.T. 2600, Australia

89. Robert E. Price, Office of Fusion Energy, G-234, Department of Energy, Washington, DC 20545

90. A. Rogister, Institute for Plasma Physics, KFA, Postfach 1913, D-5170, Jülich 1, Federal Republic of Germany

91. W. Sadowski, Office of Fusion Energy, G-234, Department of Energy, Washington, DC 20545

92. V. D. Shafranov, I. V. Kurchatov Institute of Atomic Energy, 46 Ulitsa Kurchatova, P.0. Box 3402, Moscow, U.S.S.R.

93. Yu. S. Sigov, Institute of Applied Mathematics of the U.S.S.R. Academy of Sciences, Miuskaya, Sq. 4, Moscow A-47, U.S.S.R.

94. W. M. Stacey, Jr., School of Nuclear Engineering, Georgia Institute of Technology, Atlanta, GA 30332

95. L. D. Stewart, Princeton Plasma Physics Laboratory, P.0. Box 451, Princeton, NJ 08540

96. J. B. Taylor, Culham Laboratory, U.K. Atomic Energy Authority Abingdon, 0xon, 0X14 3DB, United Kingdom

97. Thermonuclear Library, Japan Atomic Energy Research Institute, Tokai, Naka, Ibaraki, Japan

98. Francisco Verdaguer, Director, Division of Fusion, Junta de Energia Nuclear, Madrid 3, Spain

99. K. M. Zwilsky, Office of Fusion Energy, G-234, Department of Energy, Washington, DC 20545

100. Director, Research and Technical Support Division, Department of Energy, Oak Ridge Operations, P.0. Box E, Oak Ridge, TN 37830

101-335. Given distribution as shown in TID-4500, Magnetic Fusion Energy (Distribution Category UC-20a, d, and g: Plasma Systems, Fusion Systems, and Theoretical Plasma Physics) 